\title{
ANTI-SYNCHRONIZATION OF HYPERCHAOTIC PANG AND HYPERCHAOTIC WANG-CHEN SySTEMS VIA ACTIVE CONTROL
}

\author{
Sundarapandian Vaidyanathan \\ Research and Development Centre, Vel Tech Dr. RR \& Dr. SR Technical University \\ Avadi, Chennai-600 062, Tamil Nadu, INDIA \\ sundarvtu@gmail. com
}

\begin{abstract}
Hyperchaotic systems are chaotic systems having more than one positive Lyapunov exponent and they have important applications in secure data transmission and communication. This paper applies active control method for the synchronization of identical and different hyperchaotic Pang systems (2011) and hyperchaotic Wang-Chen systems (2008). Main results are proved with the stability theorems of Lypuanov stability theory and numerical simulations are plotted using MATLAB to show the synchronization of hyperchaotic systems addressed in this paper.
\end{abstract}

\section{KEYWORDS}

Anti-Synchronization, Active Control, Chaos, Hyperchaos, Hyperchaotic Systems.

\section{INTRODUCTION}

Hyperchaotic systems have a lot of important applications in several fields in Science and Engineering. They are chaotic systems with more than one positive Lyapunov exponent. The hyperchaotic systemwas first found by O.E.Rössler ([1], 1979).

Hyperchaotic systems have attractive features like high security, high capacity and high efficiency and they find miscellaneous applications in several areas like neural networks [2],oscillators [3], secure communication [4-5], encryption [6], synchronization [7], etc.

There are many important methods available in the literature for synchronization and antisynchronization like PC method [8], OGY method [9], backstepping method [10-12], sliding control method [13-15], active control method [16-18], adaptive control method [19-20], sampled-data feedback control method [21], time-delay feedback method [22], etc.

The anti-synchronization problem deals witha pair of chaotic systems called the master and slave systems, where the design goal is to anti-synchronize their states, i.e. the sum of the states of the master and slave systems approach to zero asymptotically.

This paper focuses upon active controller design for the anti-synchronization of hyperchaotic Pang systems ([23], 2011) and hyperchaotic Wang-Chen systems ([24], 2008). The main results derived in this paper were proved using Lyapunov stability theory [25].

Using active control method, new results have been derived for the anti-synchronization of identical hyperchaotic Pang systems, identical hyperchaotic Wang-Chen systems and non-identical hyperchaotic Pang and hyperchaotic Wang-Chen systems. Numerical simulations were shown using MATLAB to illustrate the main results derived in this paper. 


\section{Problem Statement}

The master system is described by the chaotic dynamics

$$
\dot{x}=A x+f(x)
$$

where $A$ is the $n \times n$ matrix of the system parameters and $f: R^{n} \rightarrow R^{n}$ is the nonlinear part.

The slave system is described by the chaotic dynamics

$$
\dot{y}=B y+g(y)+u
$$

where $B$ is the $n \times n$ matrix of the system parameters, $g: R^{n} \rightarrow R^{n}$ is the nonlinear part and $u \in R^{n}$ is the active controller to be designed.

For the pair of chaotic systems (1) and (2), the anti-synchronization problem aims to design a feedback controller $u$, which anti-synchronizes their states for all $x(0), y(0) \in R^{n}$.

Theanti-synchronization erroris defined as

$$
e=y+x
$$

Theerror dynamics is obtained as

$$
\dot{e}=B y+A x+g(y)+f(x)+u
$$

The design goal is to find a feedback controller $u$ so that

$$
\lim _{t \rightarrow \infty}\|e(t)\|=0 \text { for all } e(0) \in R^{n}
$$

Using the matrix method, we consider a candidate Lyapunov function

$V(e)=e^{T} P e$,

where $P$ is a positive definite matrix.

It is noted that $V: R^{n} \rightarrow R$ is a positive definite function.

If we find a feedback controller $u$ so that

$$
\dot{V}(e)=-e^{T} Q e,
$$

where $Q$ is a positive definite matrix, then $\dot{V}: R^{n} \rightarrow R$ is a negative definite function.

Thus, by Lyapunov stability theory [15], the error dynamics (4) is globally exponentially stable.

Hence, the states of the chaotic systems (1) and (2) will be globally and exponentially anti-synchronized for all initial conditions $x(0), y(0) \in R^{n}$. 


\section{HYPERCHAOTIC SYSTEMS}

The hyperchaotic Pang system ([23], 2011) is given by

$$
\begin{aligned}
& \dot{x}_{1}=a\left(x_{2}-x_{1}\right) \\
& \dot{x}_{2}=c x_{2}-x_{1} x_{3}+x_{4} \\
& \dot{x}_{3}=-b x_{3}+x_{1} x_{2} \\
& \dot{x}_{4}=-d\left(x_{1}+x_{2}\right)
\end{aligned}
$$

where $a, b, c, d$ are constant, positive parameters of the system.

The Pang system (8) exhibits a hyperchaotic attractor for the parametric values

$$
a=36, \quad b=3, \quad c=20, d=2
$$

The Lyapunov exponents of the system (8) for the parametric values in (9) are

$$
\lambda_{1}=1.4106, \quad \lambda_{2}=0.1232, \quad \lambda_{3}=0, \quad \lambda_{4}=-20.5339
$$

Since there are two positive Lyapunov exponents in (10), the Pang system (8) is hyperchaotic for the parametric values (9).

The phase portrait of the hyperchaotic Pang system is described in Figure 1.

The hyperchaotic Wang-Chen system ([24], 2008) is given by

$$
\begin{aligned}
& \dot{x}_{1}=\alpha\left(x_{2}-x_{1}\right)+x_{2} x_{3} \\
& \dot{x}_{2}=\gamma x_{1}-x_{1} x_{3}-x_{2}-0.5 x_{4} \\
& \dot{x}_{3}=-3 x_{3}+x_{1} x_{2} \\
& \dot{x}_{4}=\beta x_{4}+0.5 x_{1} x_{3}
\end{aligned}
$$

where $\alpha, \beta, \gamma$ are constant, positive parameters of the system.

The Wang system (11) exhibits a hyperchaotic attractor for the parametric values

$$
\alpha=40, \quad \beta=1.7, \quad \gamma=88
$$

The Lyapunov exponents of the system (9) for the parametric values in (12) are

$$
\lambda_{1}=3.2553, \quad \lambda_{2}=1.4252, \quad \lambda_{3}=0, \quad \lambda_{4}=-46.9794
$$

Since there are two positive Lyapunov exponents in (13), the Wang-Chen system (11) is hyperchaotic for the parametric values (12).

The phase portrait of the hyperchaotic Wang-Chen system is described in Figure 2. 

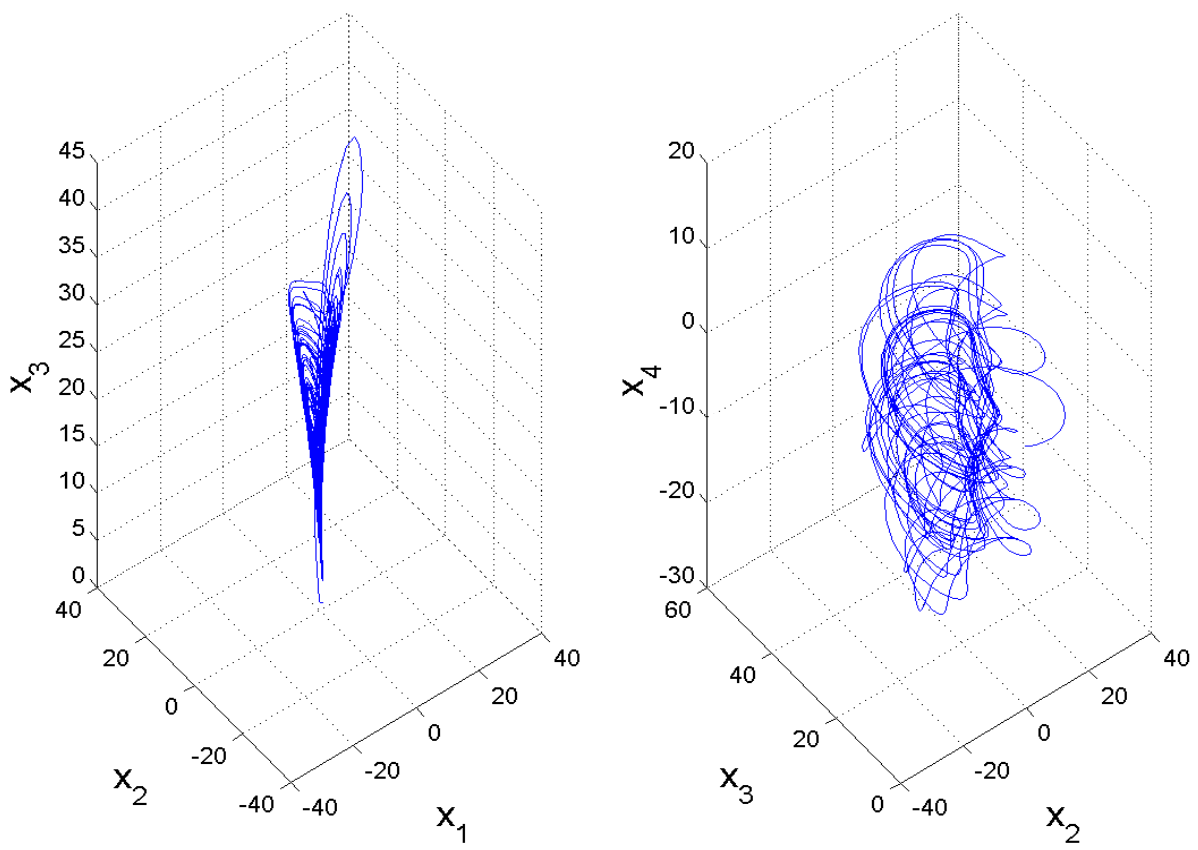

Figure 1. The Phase Portrait of the Hyperchaotic Pang System
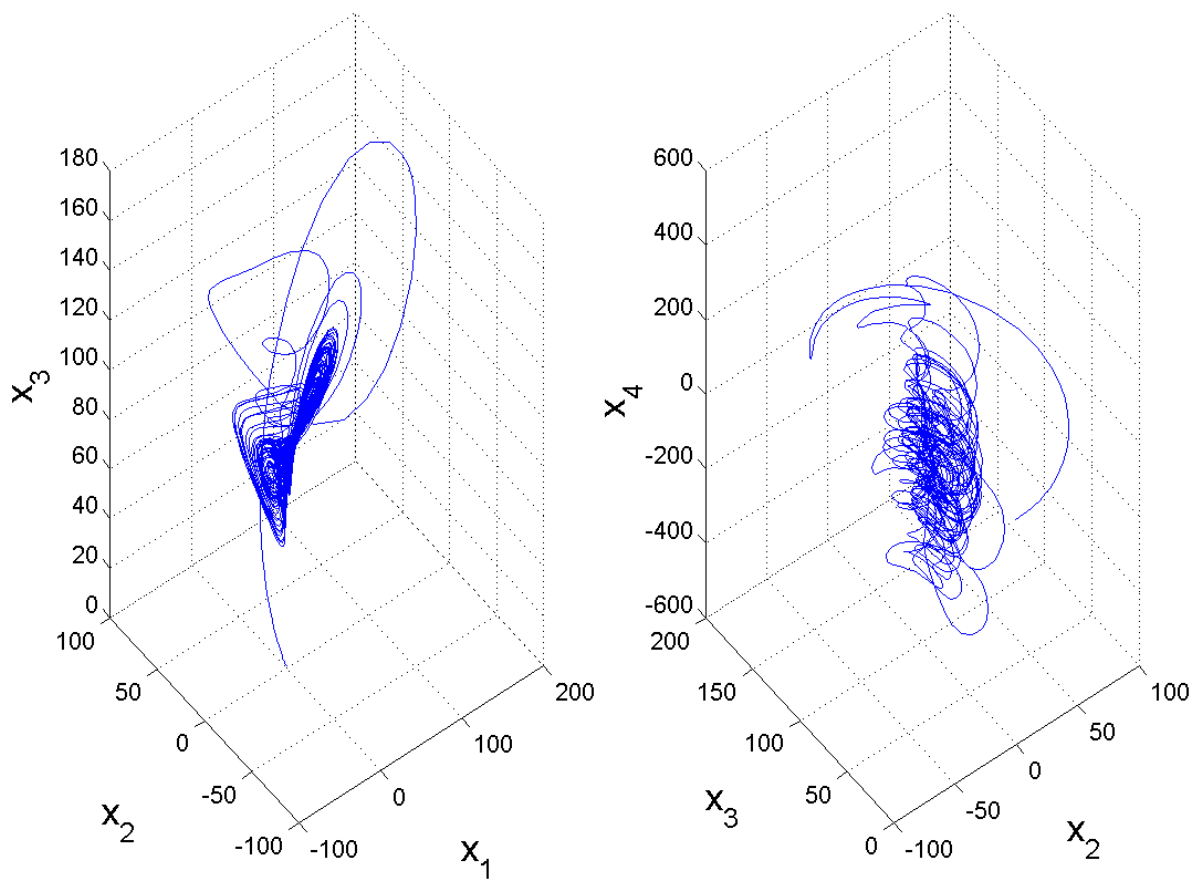

Figure 2. The Phase Portrait of the Hyperchaotic Wang-Chen System 


\section{Anti-Synchronization of Hyperchaotic Pang Systems via ACTIVE CONTROL}

In this section, we investigate the problem of anti-synchronization of two identical hyperchaotic Pang systems (2011) and derive new results via active control.

The master system is the hyperchaotic Pangsystem given by

$$
\begin{aligned}
& \dot{x}_{1}=a\left(x_{2}-x_{1}\right) \\
& \dot{x}_{2}=c x_{2}-x_{1} x_{3}+x_{4} \\
& \dot{x}_{3}=-b x_{3}+x_{1} x_{2} \\
& \dot{x}_{4}=-d\left(x_{1}+x_{2}\right)
\end{aligned}
$$

where $a, b, c, d$ are positive parameters of the system and $x \in R^{4}$ is the state.

The slave system is the controlled hyperchaotic Pangsystem given by

$$
\begin{aligned}
& \dot{y}_{1}=a\left(y_{2}-y_{1}\right)+u_{1} \\
& \dot{y}_{2}=c y_{2}-y_{1} y_{3}+y_{4}+u_{2} \\
& \dot{y}_{3}=-b y_{3}+y_{1} y_{2}+u_{3} \\
& \dot{y}_{4}=-d\left(y_{1}+y_{2}\right)+u_{4}
\end{aligned}
$$

where $y \in R^{4}$ is the state and $u_{1}, u_{2}, u_{3}, u_{4}$ are the active controllers to be designed.

For the anti-synchronization, the error $e$ is defined as

$$
e_{i}=y_{i}+x_{i}, \quad(i=1,2,3,4)
$$

We obtain the error dynamics as

$$
\begin{aligned}
& \dot{e}_{1}=a\left(e_{2}-e_{1}\right)+u_{1} \\
& \dot{e}_{2}=c e_{2}+e_{4}-y_{1} y_{3}-x_{1} x_{3}+u_{2} \\
& \dot{e}_{3}=-b e_{3}+y_{1} y_{2}+x_{1} x_{2}+u_{3} \\
& \dot{e}_{4}=-d\left(e_{1}+e_{2}\right)+u_{4}
\end{aligned}
$$

The active controller to achieve anti-synchronization is chosen as

$$
\begin{aligned}
& u_{1}=-a\left(e_{2}-e_{1}\right)-k_{1} e_{1} \\
& u_{2}=-c e_{2}-e_{4}+y_{1} y_{3}+x_{1} x_{3}-k_{2} e_{2} \\
& u_{3}=b e_{3}-y_{1} y_{2}-x_{1} x_{2}-k_{3} e_{3} \\
& u_{4}=d\left(e_{1}+e_{2}\right)-k_{4} e_{4}
\end{aligned}
$$

where $k_{i},(i=1,2,3,4)$ are positive gains.

By the substitution of (18) into (17), the error dynamics is simplified as 


$$
\dot{e}_{i}=-k_{i} e_{i}, \quad(i=1,2,3,4)
$$

Thus, we obtain the following result.

Theorem 4.1 The nonlinear controller defined by Eq. (18) achieves global and exponential antisynchronization of the identical hyperchaotic Pang systems (14) and (15) for all initial conditions $x(0), y(0) \in R^{4}$.

Proof.The proof is via Lyapunov stability theorem [25] for global exponential stability.

We take the quadratic Lyapunov function

$$
V(e)=\frac{1}{2} e^{T} e=\frac{1}{2}\left(e_{1}^{2}+e_{2}^{2}+e_{3}^{2}+e_{4}^{2}\right),
$$

which is a positive definite function on $R^{4}$.

When we differentiate (18) along the trajectories of (17), we get

$$
\dot{V}(e)=-k_{1} e_{1}^{2}-k_{2} e_{2}^{2}-k_{3} e_{3}^{2}-k_{4} e_{4}^{2}
$$

which is a negative definite function on $R^{4}$. Hence, the error dynamics (19) is globally exponentially stable for all $e(0) \in R^{4}$. This completes the proof.

Next, we illustrate our anti-synchronization results with MATLAB simulations. The $4^{\text {th }}$ order Runge-Kutta method with time-step $h=10^{-8}$ has been applied to solve the hyperchaotic Pang systems (14) and (15) with the active nonlinear controller defined by (18).

The feedback gains in the active controller (18) are taken as $k_{i}=5,(i=1,2,3,4)$.

The parameters of the hyperchaotic Pang systems are taken as in the hyperchaotic case, i.e.

$$
a=36, \quad b=3, c=20, d=2
$$

For simulations, the initial conditions of the master system (14) are taken as

$$
x_{1}(0)=12, \quad x_{2}(0)=20, \quad x_{3}(0)=-14, \quad x_{4}(0)=-27
$$

Also, the initial conditions of the slave system (15) are taken as

$$
y_{1}(0)=32, \quad y_{2}(0)=15, \quad y_{3}(0)=20, \quad y_{4}(0)=-10
$$

Figure 3 depicts the anti-synchronization of the identical hyperchaotic Pang systems.

Figure 4 depicts the time-history of the anti-synchronization errors $e_{1}, e_{2}, e_{3}, e_{4}$. 

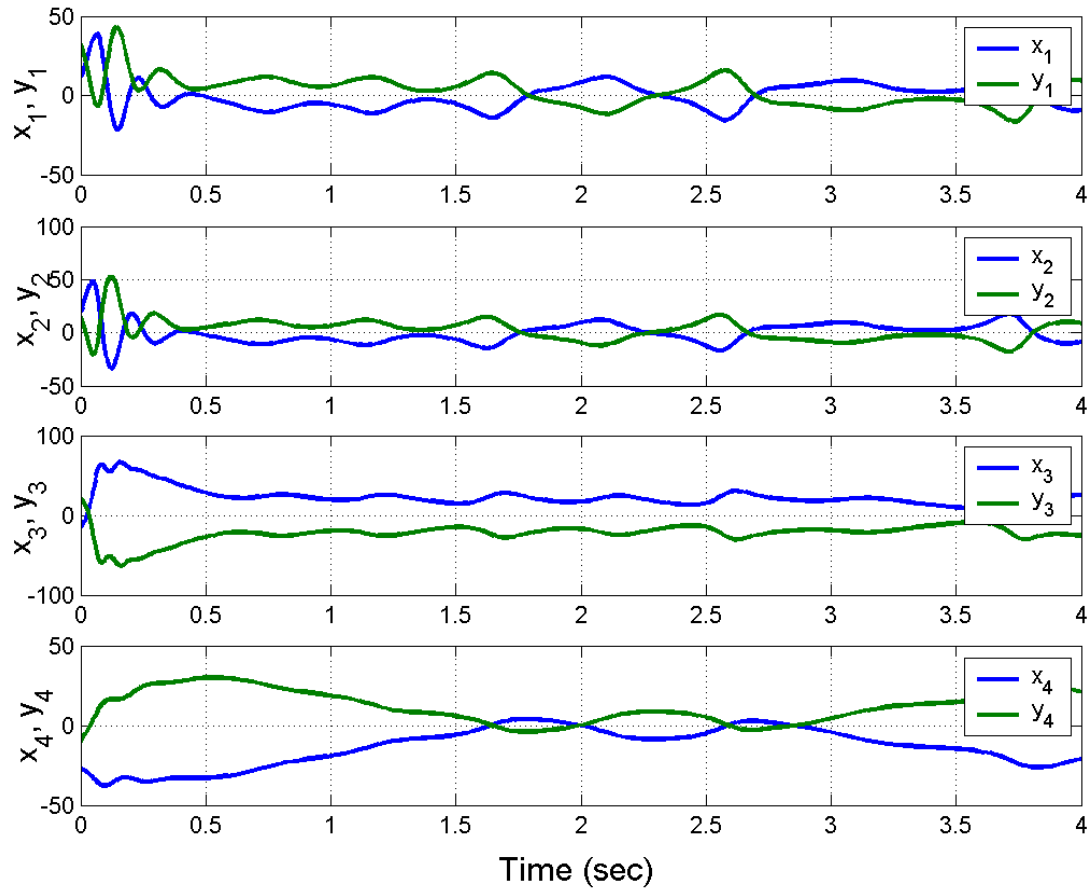

Figure 3. Anti-Synchronization of Identical Hyperchaotic Pang Systems

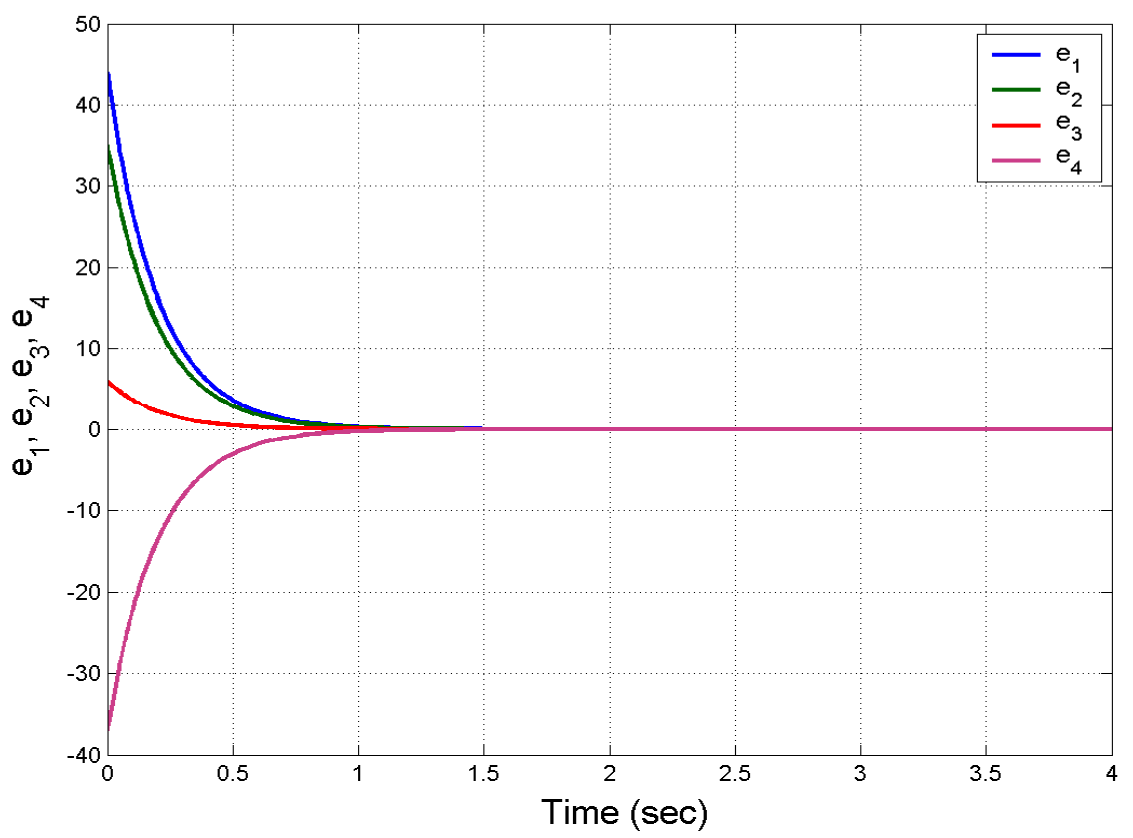

Figure 4. Time-History of the Anti-Synchronization Errors $e_{1}, e_{2}, e_{3}, e_{4}$ 


\section{ANTI-SynChronization OF HyPERChAotic WANG-CHEN SySTEMS Via ACTIVE CONTROL}

In this section, we investigate the problem of anti-synchronization of two identical hyperchaotic Wang-Chen systems (2008) and derive new results via active control.

The master system is the hyperchaotic Wang-Chensystem given by

$$
\begin{aligned}
& \dot{x}_{1}=\alpha\left(x_{2}-x_{1}\right)+x_{2} x_{3} \\
& \dot{x}_{2}=\gamma x_{1}-x_{1} x_{3}-x_{2}-0.5 x_{4} \\
& \dot{x}_{3}=-3 x_{3}+x_{1} x_{2} \\
& \dot{x}_{4}=\beta x_{4}+0.5 x_{1} x_{3}
\end{aligned}
$$

where $\alpha, \beta, \gamma$ are positive parameters of the system and $x \in R^{4}$ is the state.

The slave system is the controlled hyperchaotic Wang-Chensystem given by

$$
\begin{aligned}
& \dot{y}_{1}=\alpha\left(y_{2}-y_{1}\right)+y_{2} y_{3}+u_{1} \\
& \dot{y}_{2}=\gamma y_{1}-y_{1} y_{3}-y_{2}-0.5 y_{4}+u_{2} \\
& \dot{y}_{3}=-3 y_{3}+y_{1} y_{2}+u_{3} \\
& \dot{y}_{4}=\beta y_{4}+0.5 y_{1} y_{3}+u_{4}
\end{aligned}
$$

where $y \in R^{4}$ is the state and $u_{1}, u_{2}, u_{3}, u_{4}$ are the active controllers to be designed.

For the anti-synchronization, the error $e$ is defined as

$$
e_{i}=y_{i}+x_{i}, \quad(i=1,2,3,4)
$$

We obtain the error dynamics as

$$
\begin{aligned}
& \dot{e}_{1}=\alpha\left(e_{2}-e_{1}\right)+y_{2} y_{3}+x_{2} x_{3}+u_{1} \\
& \dot{e}_{2}=\gamma e_{1}-e_{2}-0.5 e_{4}-y_{1} y_{3}-x_{1} x_{3}+u_{2} \\
& \dot{e}_{3}=-3 e_{3}+y_{1} y_{2}+x_{1} x_{2}+u_{3} \\
& \dot{e}_{4}=\beta e_{4}+0.5\left(y_{1} y_{3}+x_{1} x_{3}\right)+u_{4}
\end{aligned}
$$

The active controller to achieve anti-synchronization is chosen as

$$
\begin{aligned}
& u_{1}=-\alpha\left(e_{2}-e_{1}\right)-y_{2} y_{3}-x_{2} x_{3}-k_{1} e_{1} \\
& u_{2}=-\gamma e_{1}+e_{2}+0.5 e_{4}+y_{1} y_{3}+x_{1} x_{3}-k_{2} e_{2} \\
& u_{3}=3 e_{3}-y_{1} y_{2}-x_{1} x_{2}-k_{3} e_{3} \\
& u_{4}=-\beta e_{4}-0.5\left(y_{1} y_{3}+x_{1} x_{3}\right)-k_{4} e_{4}
\end{aligned}
$$

where $k_{i},(i=1,2,3,4)$ are positive gains. 
By the substitution of (26) into (25), the error dynamics is simplified as

$$
\dot{e}_{i}=-k_{i} e_{i}, \quad(i=1,2,3,4)
$$

Thus, we obtain the following result.

Theorem 5.1 The nonlinear controller defined by Eq. (26) achieves global and exponential antisynchronization of the identical hyperchaotic Wang-Chensystems (22) and (23) for all initial conditions $x(0), y(0) \in R^{4}$.

Proof.The proof is via Lyapunov stability theorem [25] for global exponential stability.

We take the quadratic Lyapunov function

$$
V(e)=\frac{1}{2} e^{T} e=\frac{1}{2}\left(e_{1}^{2}+e_{2}^{2}+e_{3}^{2}+e_{4}^{2}\right)
$$

which is a positive definite function on $R^{4}$.

When we differentiate (26) along the trajectories of (25), we get

$$
\dot{V}(e)=-k_{1} e_{1}^{2}-k_{2} e_{2}^{2}-k_{3} e_{3}^{2}-k_{4} e_{4}^{2}
$$

which is a negative definite function on $R^{4}$. Hence, the error dynamics (27) is globally exponentially stable for all $e(0) \in R^{4}$. This completes the proof.

Next, we illustrate our anti-synchronization results with MATLAB simulations. The $4^{\text {th }}$ order Runge-Kutta method with time-step $h=10^{-8}$ has been applied to solve the hyperchaotic WangChen systems (22) and (23) with the active nonlinear controller defined by (26).

The feedback gains in the active controller (26) are taken as $k_{i}=5,(i=1,2,3,4)$.

The parameters of the hyperchaotic WCsystems are taken as in the hyperchaotic case, i.e.

$$
\alpha=40, \quad \beta=1.7, \quad \gamma=88
$$

For simulations, the initial conditions of the master system (22) are taken as

$$
x_{1}(0)=-7, \quad x_{2}(0)=12, \quad x_{3}(0)=-15, \quad x_{4}(0)=17
$$

Also, the initial conditions of the slave system (23) are taken as

$$
y_{1}(0)=30, \quad y_{2}(0)=25, \quad y_{3}(0)=-18, \quad y_{4}(0)=-5
$$

Figure 5depicts the anti-synchronization of the identical hyperchaotic Wang-Chen systems. Figure 6depicts the time-history of the anti-synchronization errors $e_{1}, e_{2}, e_{3}, e_{4}$. 

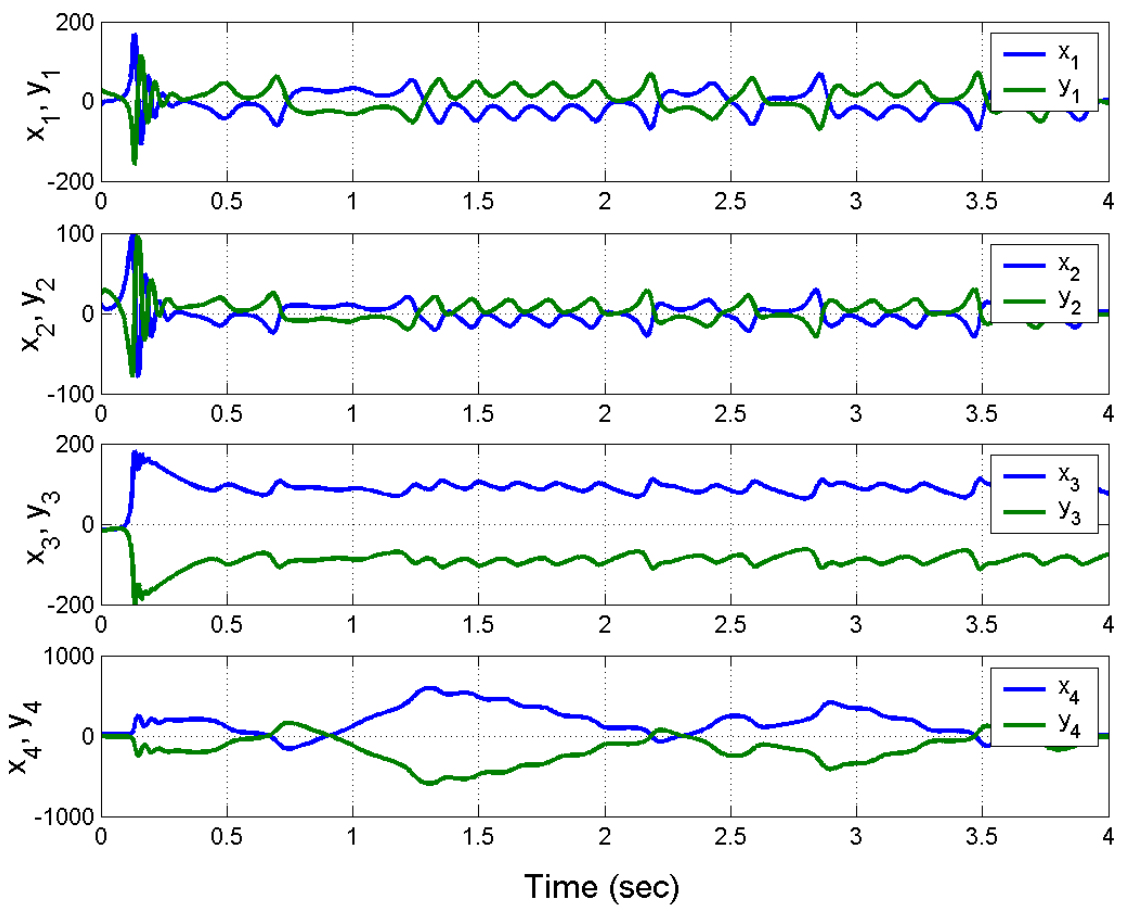

Figure 5. Anti-Synchronization of Identical Hyperchaotic Wang-Chen Systems

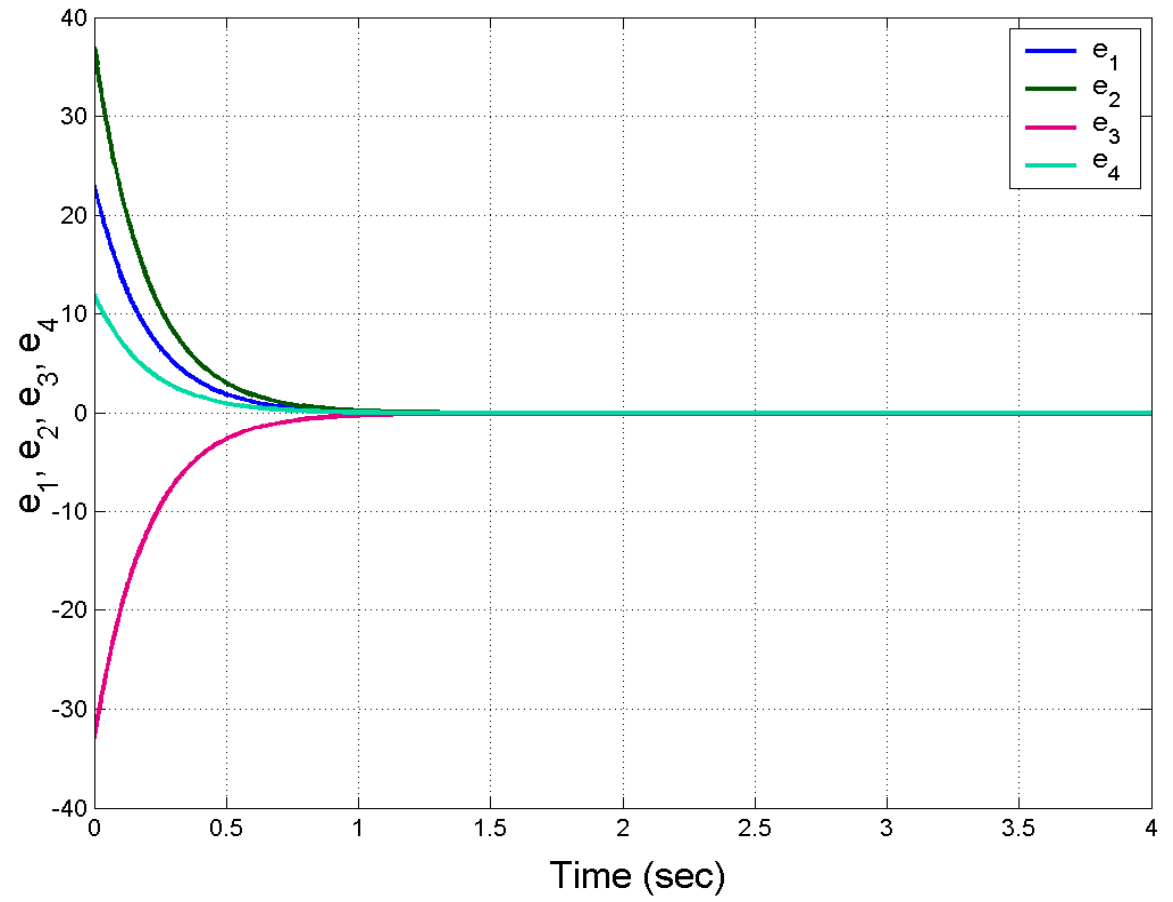

Figure 6. Time-History of the Anti-Synchronization Errors $e_{1}, e_{2}, e_{3}, e_{4}$ 


\section{Anti-Synchronization Of Hyperchaotic Pang and Hyperchaotic Wang- Chen Systems via Active Control}

In this section, we derive new results for the problem of anti-synchronization of hyperchaotic Pang (2011) and hyperchaotic Wang-Chen systems (2008) via active control.

The master system is the hyperchaotic Pang system given by

$$
\begin{aligned}
& \dot{x}_{1}=a\left(x_{2}-x_{1}\right) \\
& \dot{x}_{2}=c x_{2}-x_{1} x_{3}+x_{4} \\
& \dot{x}_{3}=-b x_{3}+x_{1} x_{2} \\
& \dot{x}_{4}=-d\left(x_{1}+x_{2}\right)
\end{aligned}
$$

where $a, b, c, d$ are positive parameters of the system and $x \in R^{4}$ is the state. The slave system is the controlled hyperchaotic Wang-Chensystem given by

$$
\begin{aligned}
& \dot{y}_{1}=\alpha\left(y_{2}-y_{1}\right)+y_{2} y_{3}+u_{1} \\
& \dot{y}_{2}=\gamma y_{1}-y_{1} y_{3}-y_{2}-0.5 y_{4}+u_{2} \\
& \dot{y}_{3}=-3 y_{3}+y_{1} y_{2}+u_{3} \\
& \dot{y}_{4}=\beta y_{4}+0.5 y_{1} y_{3}+u_{4}
\end{aligned}
$$

where $\alpha, \beta, \gamma$ are positive parameters, $y \in R^{4}$ is the state and $u_{1}, u_{2}, u_{3}, u_{4}$ are the active controllers to be designed.

For the anti-synchronization, the error $e$ is defined as

$$
e_{i}=y_{i}+x_{i}, \quad(i=1,2,3,4)
$$

We obtain the error dynamics as

$$
\begin{aligned}
& \dot{e}_{1}=\alpha\left(y_{2}-y_{1}\right)+a\left(x_{2}-x_{1}\right)+y_{2} y_{3}+u_{1} \\
& \dot{e}_{2}=\gamma y_{1}-y_{2}-0.5 y_{4}+c x_{2}+x_{4}-y_{1} y_{3}-x_{1} x_{3}+u_{2} \\
& \dot{e}_{3}=-3 y_{3}-b x_{3}+y_{1} y_{2}+x_{1} x_{2}+u_{3} \\
& \dot{e}_{4}=\beta y_{4}-d\left(x_{1}+x_{2}\right)+0.5 y_{1} y_{3}+u_{4}
\end{aligned}
$$

The active controller to achieve anti-synchronization is chosen as

$$
\begin{aligned}
& u_{1}=-\alpha\left(y_{2}-y_{1}\right)-a\left(x_{2}-x_{1}\right)-y_{2} y_{3}-k_{1} e_{1} \\
& u_{2}=-\gamma y_{1}+y_{2}+0.5 y_{4}-c x_{2}-x_{4}+y_{1} y_{3}+x_{1} x_{3}-k_{2} e_{2} \\
& u_{3}=3 y_{3}+b x_{3}-y_{1} y_{2}-x_{1} x_{2}-k_{3} e_{3} \\
& u_{4}=-\beta y_{4}+d\left(x_{1}+x_{2}\right)-0.5 y_{1} y_{3}-k_{4} e_{4}
\end{aligned}
$$

where $k_{i},(i=1,2,3,4)$ are positive gains. 
By the substitution of (34) into (33), the error dynamics is simplified as

$$
\dot{e}_{i}=-k_{i} e_{i}, \quad(i=1,2,3,4)
$$

Thus, we obtain the following result.

Theorem 6.1 The nonlinear controller defined by Eq. (34) achieves global and exponential antisynchronization of the non-identical hyperchaotic Pang system (30) and the controlled hyperchaotic Wang-Chen system (31) for all initial conditions $x(0), y(0) \in R^{4}$.

Proof.The proof is via Lyapunov stability theorem [25] for global exponential stability.

We take the quadratic Lyapunov function

$$
V(e)=\frac{1}{2} e^{T} e=\frac{1}{2}\left(e_{1}^{2}+e_{2}^{2}+e_{3}^{2}+e_{4}^{2}\right)
$$

which is a positive definite function on $R^{4}$.

When we differentiate (34) along the trajectories of (33), we get

$$
\dot{V}(e)=-k_{1} e_{1}^{2}-k_{2} e_{2}^{2}-k_{3} e_{3}^{2}-k_{4} e_{4}^{2}
$$

which is a negative definite function on $R^{4}$. Hence, the error dynamics (35) is globally exponentially stable for all $e(0) \in R^{4}$. This completes the proof.

Next, we illustrate our anti-synchronization results with MATLAB simulations. The $4^{\text {th }}$ order Runge-Kutta method with time-step $h=10^{-8}$ has been applied to solve the hyperchaotic systems (30) and (31) with the active nonlinear controller defined by (34).

The feedback gains in the active controller (34) are taken as $k_{i}=5,(i=1,2,3,4)$.

The parameters of the hyperchaotic Pang and hyperchaotic WC systems are taken as in the hyperchaotic case, i.e.

$$
a=36, \quad b=3, \quad c=20, \quad d=2, \quad \alpha=40, \quad \beta=1.7, \quad \gamma=88
$$

For simulations, the initial conditions of the master system (30) are taken as

$$
x_{1}(0)=27, \quad x_{2}(0)=-16, \quad x_{3}(0)=-12, \quad x_{4}(0)=31
$$

Also, the initial conditions of the slave system (31) are taken as

$$
y_{1}(0)=10, \quad y_{2}(0)=5, \quad y_{3}(0)=-28, \quad y_{4}(0)=-14
$$

Figure 7depicts the anti-synchronization of the hyperchaotic Pang and Wang-Chen systems.

Figure 8depicts the time-history of the anti-synchronization errors $e_{1}, e_{2}, e_{3}, e_{4}$. 

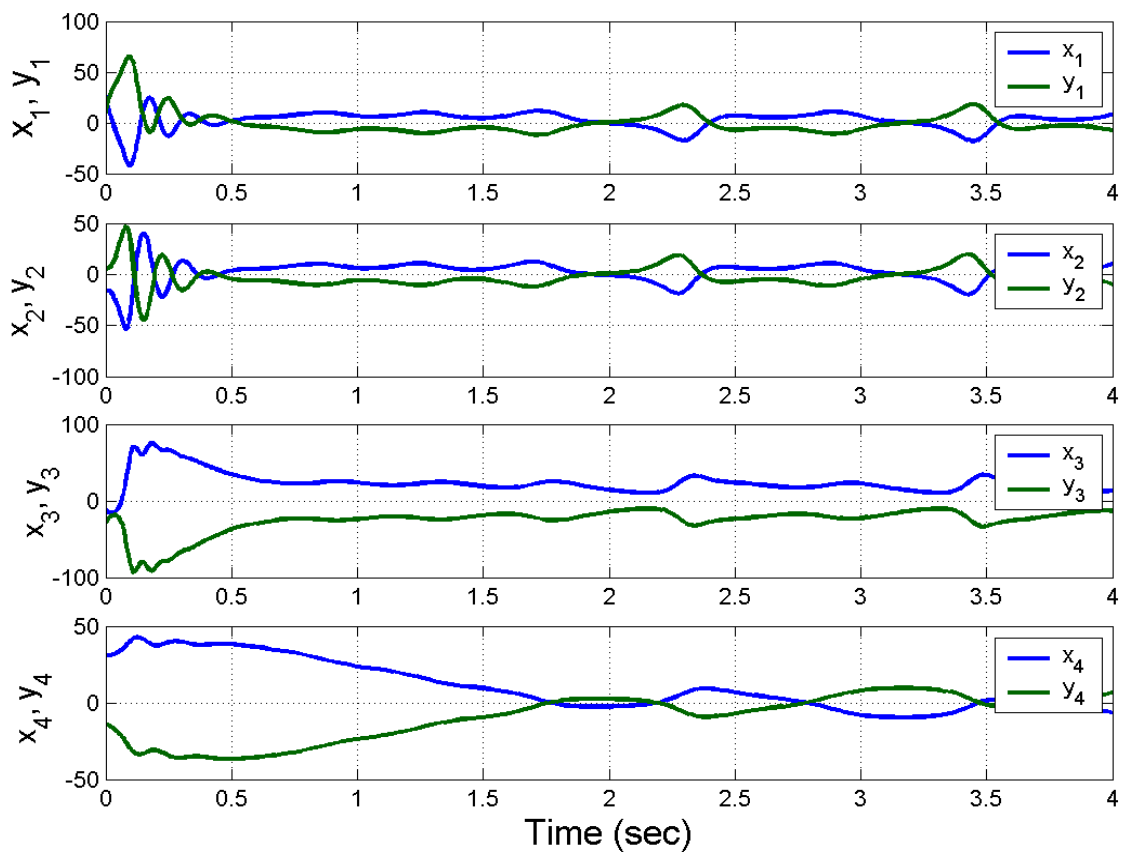

Figure 7. Anti-Synchronization of Identical Hyperchaotic Pang and Hyperchaotic WC Systems

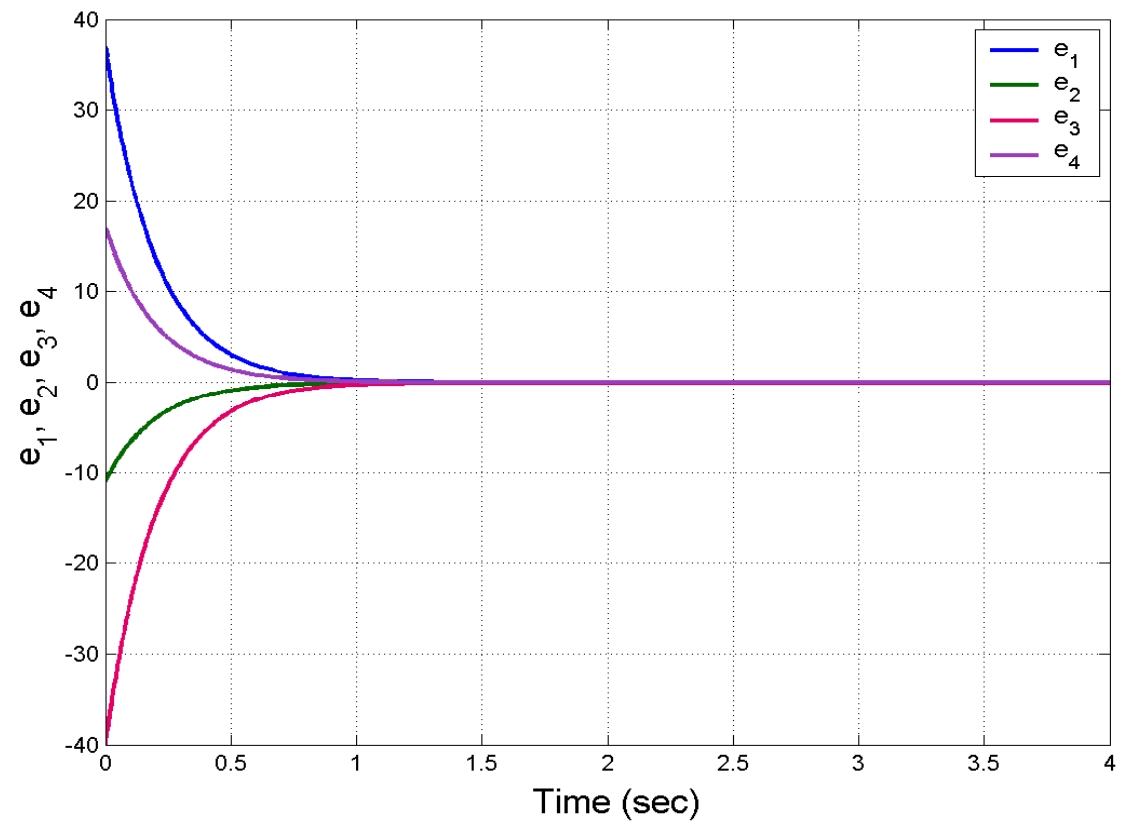

Figure 8. Time-History of the Anti-Synchronization Errors $e_{1}, e_{2}, e_{3}, e_{4}$ 


\section{Conclusions}

This paper derived new results for the anti-synchronization of hyperchaotic Pang systems (2011) and hyperchaotic Wang-Chen systems (2008) using active control method. Explicitly, active control laws were derived for globally anti-synchronizing the states of identical hyperchaotic Pang systems, identical hyperchaotic Wang-Chen systems and non-identical hyperchaotic Pang and Wang-Chen systems. The main results validating the anti-synchronizing active controllers were proved using Lyapunov stability theory. MATLAB simulations were shown to illustrate the anti-synchronization results derived in this paper for hyperchaotic Pang and hyperchaotic WangChen systems.

\section{REFERENCES}

[1] Rössler, O.E. (1979) “An equation for hyperchaos,” Physics Letters A, Vol. 71, pp 155-157.

[2] Huang, Y. \& Yang, X.S. (2006) "Hyperchaos and bifurcation in a new class of four-dimensional Hopfield neural networks," Neurocomputing, Vol. 69, pp 13-15.

[3] Machado, L.G., Savi, M.A. \& Pacheco, P.M.C.L. (2003) "Nonlinear dynamics and chaos in coupled shape memory oscillators," International Journal of Solids and Structures, Vol. 40, No. 19, pp. 5139-5156.

[4] Tao, Y. (1999) "Chaotic secure communication systems - history and new results", Telecommun. Review, Vol. 9, pp 597-634.

[5] Li, C., Liao, X. \& Wong, K.W. (2005) "Lag synchronization of hyperchaos with applications to secure communications," Chaos, Solitons \& Fractals, Vol. 23, No. 1, pp 183-193.

[6] Prokhorov, M.D. \& Ponomarenko, V.I. (2008) "Encryption and decryption of information in chaotic communication systems governed by delay-differential equations," Chaos, Solitons \& Fractals, Vol. 35, No. 5, pp 871-877.

[7] Yassen, M.T. (2008) "Synchronization hyperchaos of hyperchaotic systems", Chaos, Solitons and Fractals, Vol. 37, pp 465-475.

[8] Pecora, L.M. \& Carroll, T.L. (1990) "Synchronization in chaotic systems", Phys. Rev. Lett., Vol. 64, pp 821-824.

[9] Ott, E., Grebogi, C. \& Yorke, J.A. (1990) "Controlling chaos”, Phys. Rev. Lett., Vol. 64, pp 11961199.

[10] Bowong, S. \& Kakmeni, F.M.M. (2004) "Synchronization of uncertain chaotic systems via backstepping approach,” Chaos, Solitons \& Fractals, Vol. 21, No. 4, pp 999-1011.

[11] Suresh, R, \& Sundarapandian, V. (2012) "Global chaos synchronization of WINDMI and Coullet chaotic systems by backstepping control”, Far East J. Math. Sciences, Vol. 67, No. 2, pp 265-287.

[12] Suresh, R. \& Sundarapandian, V. (2012) "Hybrid synchronization of n-scroll Chua and Lur'e chaotic systems via backstepping control with novel feedback", Arch. Control Sciences, Vol. 22, No. 3, pp 255-278.

[13] Senejohnny, D.M. \& Delavari, H. (2012) "Active sliding observer scheme based fractional chaos synchronization,” Comm. Nonlinear Sci. Numerical Simulation, Vol. 17, No. 11, pp 4373-4383.

[14] Sundarapandian, V. (2012) "Anti-synchronization of hyperchaotic Xu systems via sliding mode control”, International Journal of Embedded Systems, Vol. 2, No. 2, pp 51-61.

[15] Sundarapandian, V. (2013) "Anti-synchronizing sliding controller design for identical Pan systems," International Journal of Computational Science and Information Technology, Vol. 1, No. 1, pp 1-9.

[16] Huang, L. Feng, R. \& Wang, M. (2004) "Synchronization of chaotic systems via nonlinear control," Physics Letters A, Vol. 320, No. 4, pp 271-275.

[17] Lei, Y., Xu, W. \& Zheng, H. (2005) "Synchronization of two chaotic nonlinear gyros using active control," Physics Letters A, Vol. 343, pp 153-158.

[18] Sarasu, P. \& Sundarapandian, V. (2011) "Active controller design for generalized projective synchronization of four-scroll chaotic systems", International Journal of System Signal Control and Engineering Application, Vol. 4, No. 2, pp 26-33.

[19] Sundarapandian, V. (2012) "Adaptive control and synchronization of a generalized Lotka-Volterra system," Vol. 1, No. 1, pp 1-12.

[20] Sundarapandian, V. (2013) "Adaptive controller and synchronizer design for hyperchaotic Zhou system with unknown parameters,” Vol. 1, No. 1, pp 18-32. 
International Journal of Control Theory and Computer Modeling (IJCTCM) Vol.3, No.2, March 2013

[21] Zhao, J. \& Lü, J. (2006) "Using sampled-data feedback control and linear feedback synchronization in a new hyperchaotic system," Chaos, Solitons \& Fractals, Vol. 35, pp. 376-382.

[22] Ma, H., Deshmukh, V., Butcher, E. \& Averina, V. (2005) "Delayed state feedback and chaos control for time-periodic systems via a symbolic approach", Communications in Nonlinear Science and Numerical Simulation, Vol. 10, No. 5, pp 479-497.

[23] Pang, S. \& Liu, Y. (2011) "A new hyperchaotic system from the Lü system and its control," Journal of Computational and Applied Mathematics, Vol. 235, pp 2775-2789.

[24] Wang, J. \& Chen, Z. (2008) "A novel hyperchaotic system and its complex dynamics," International Journal of Bifurcation and Chaos, Vol. 18, No. 11, pp 3309-3324.

[25] Hahn, W. (1967) The Stability of Motion, Springer, Berlin.

\section{Author}

Dr. V. Sundarapandian earned his D.Sc. in Electrical and Systems Engineering from Washington University, St. Louis, USA in May 1996. He is Professor and Dean of the R \& D Centre at Vel Tech Dr. RR \& Dr. SR Technical University, Chennai, Tamil Nadu, India. So far, he has published over 300 research works in refereed international journals. He has also published over 200 research papers in National and International Conferences. He has delivered Key Note Addresses at many International Conferences with IEEE and Springer Proceedings. He is an India Chair of AIRCC. He is the Editor-inChief of the AIRCC Control Journals -International Journal of Instrumentation

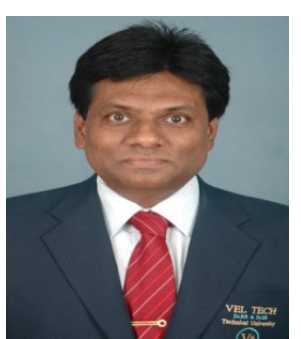
and Control Systems, International Journal of Control Theory and Computer Modeling, International Journal of Information Technology, Control and Automation, International Journal of Chaos, Control, Modelling and Simulation and International Journal of Information Theory, Modeling and Computing. His research interests are Control Systems, Chaos Theory, Soft Computing, Operations Research, Mathematical Modelling and Scientific Computing. He has published four text-books and conducted many workshops on Scientific Computing, MATLAB and SCILAB. 